\title{
Adaptive Pilot Signaling in the Uplink of OFDM- Based Wireless Systems
}

\author{
Cheong-Hwan Kim and Yong-Hwan Lee \\ School of Electrical Engineering and INMC \\ Seoul National University \\ Kwanak P. O. Box 34, Seoul 151-600 Korea \\ e-mail: kkins@ttl.snu.ac.kr, ylee@snu.ac.kr
}

\begin{abstract}
Orthogonal frequency division multiplexing (OFDM) has been considered as one of key transmission technologies for high-speed wireless communication systems. For channel-adaptive transmission of OFDM signal, base stations transmit a common pilot signal in the downlink, but mobile stations transmit individual pilot signal in the uplink. As a consequence, the uplink may suffer from poorer channel estimation accuracy than the downlink. This problem can be alleviated by increasing the pilot signal density. But this reduces the time and frequency resources for the data transmission, deteriorating the actual transmission throughput. In this paper, we propose an adaptive pilot signaling scheme that adjusts the pilot density in response to the change of channel characteristics. Simulation results show that the proposed scheme can remarkably reduce the pilot overhead without performance degradation, compared to conventional schemes.
\end{abstract}

\section{INTRODUCTION}

In recent years, orthogonal frequency division multiplexing (OFDM) has attracted much attention as an effective modulation technique for next generation wireless communication systems [1], [2]. It can provide high spectral efficiency and mitigate inter-symbol interference (ISI) in frequency selective fading channel. However, this may be achievable with the use of accurate channel information, which is not easily obtainable.

For the channel estimation, a known signal so-called pilot signal is usually employed [3]-[5]. In the downlink, the base station (BS) transmits a common pilot signal which spans the whole channel bandwidth, enabling to get affordable estimation accuracy. In the uplink, however, it may not be feasible for each mobile station (MS) to use such a full-band pilot signal mainly due to limited overhead budget. This may cause the uplink performance to suffer from inaccurate channel information. This problem can be alleviated by increasing the pilot signal density. However, this may reduce the resource for the data transmission in time and/or frequency domain, decreasing the overall throughput.

It is well known that wireless channel has characteristics correlated in the time and/or frequency domain, which affects the performance of channel estimation. For example, when the channel gain is loosely correlated (or equivalently the channel is highly selective in time or frequency), the accuracy of the channel estimation may significantly deteriorate. In conventional systems, MSs transmit the pilot signal with a fixed density which is designed to provide acceptable estimation accuracy even in the worst channel condition at the expense of increased overhead. However, when the channel condition is mild, this may provide estimation accuracy much more than being required, which is not desirable in terms of the resource allocation for the pilot signal. In this paper, we propose an adaptive pilot scheme for the uplink that adjusts the density of pilot signal in response to the change of channel condition. By exploiting the channel characteristics such as the Doppler frequency and delay spread, the proposed scheme minimizes the pilot density while maintaining desired channel estimation accuracy.

This paper is organized as follows. Section II describes the system model in consideration. In Section III, the proposed pilot scheme is described. The performance of the proposed scheme is verified by computer simulation in Section IV. Finally, conclusions are given in Section V.

\section{SySTEM MODELING}

Consider the uplink of an OFDM-based wireless access system, where the received signal at the $n$-th OFDM symbol and the $k$-th subcarrier is represented as

$$
Y(n, k)=H(n, k) X(n, k)+N(n, k) .
$$

Here, $X(n, k)$ is the transmitted symbol from the MS, $H(n, k)$ is the response of the channel and $N(n, k)$ denotes additive white Gaussian noise (AWGN) with variance $\sigma_{n}^{2}$. We assume that each MS uses a signal transmission segment comprising $N_{t}$ symbols and $N_{f}$ subcarriers. As illustrated in Fig. 1, the MS transmits pilot symbols scattered in the time and frequency domain over the signal transmission segment in a regular pattern [6]. In this paper we consider the use of a rectangle pattern for the pilot signal placement, which can provide nearly optimum performance while requiring low computational complexity [6]. We assume that each pilot symbol is transmitted every $d_{t}+1$ OFDM symbols and every $d_{f}+1$ subcarriers in the signal transmission segment as shown in Fig. 1, where $T_{s}$ and $\Delta f$ denote the OFDM symbol duration and the subcarrier spacing, respectively. 


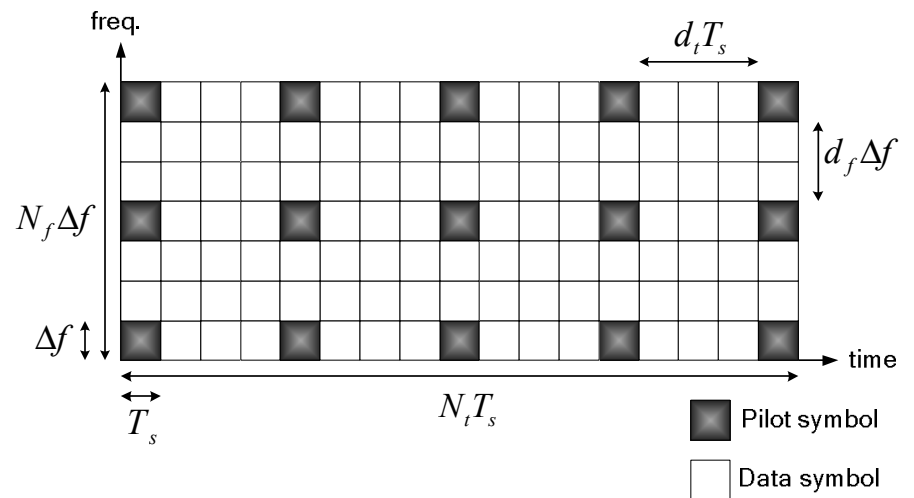

Fig. 1. Signal transmission segment in the uplink.

Let $r_{H}(\Delta n, \Delta k)$ be the joint correlation function of $H(n, k)$ in the time and frequency domain defined by

$$
r_{H}(\Delta n, \Delta k)=E\left\{H(n+\Delta n, k+\Delta k) H^{*}(n, k)\right\}
$$

where $E\{X\}$ denotes the expectation of random variable $X$ and the superscript * denotes complex conjugate. When the channel has multiple paths uncorrelated to each other, the joint correlation function can be represented as [6]

$$
r_{H}(\Delta n, \Delta k)=r_{t}(\Delta n) r_{f}(\Delta k)
$$

where $r_{t}(\Delta n)$ and $r_{f}(\Delta k)$ denote the correlation function in the time and frequency domain, respectively, defined by

$$
\begin{gathered}
r_{t}(\Delta n)=E\left\{H(n+\Delta n, k) H^{*}(n, k)\right\} \\
r_{f}(\Delta k)=E\left\{H(n, k+\Delta k) H^{*}(n, k)\right\} .
\end{gathered}
$$

Assuming that the channel has a power delay profile exponentially decaying, (4) and (5) can be represented as [6], [7]

$$
\begin{gathered}
r_{t}(\Delta n)=J_{0}\left(2 \pi f_{d} \Delta n\right), \\
r_{f}(\Delta k)=2 \sum_{l=0}^{L-1} \cos \frac{2 \pi \Delta k}{L} e^{-\frac{\tau_{o}}{\tau_{m m s}} l}+j 2 \sum_{l=0}^{L-1} \sin \frac{2 \pi \Delta k}{L} e^{-\frac{\tau_{o}}{\tau_{m m s}} l}
\end{gathered}
$$

where $J_{0}(x)$ is the zero-th order Bessel function of the first kind, $f_{d}$ denotes the Doppler frequency which is linearly proportional to the MS mobility, $L$ is the number of multipath, $\tau_{o}$ is the time duration between the taps and $\tau_{r m s}$ denotes the root mean squared (RMS) delay spread determined by $[8]$

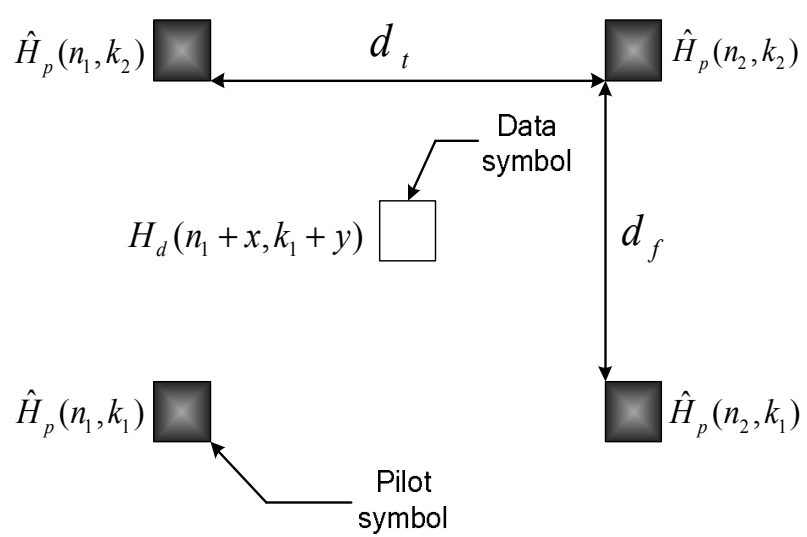

Fig. 2. Channel estimation using pilot signal.

$$
\tau_{r m s}=\sqrt{\frac{\sum_{l=0}^{L-1} \sigma_{l}^{2} \tau_{l}^{2}}{\sum_{l=0}^{L-1} \sigma_{l}^{2}}-\left(\frac{\sum_{l=0}^{L-1} \sigma_{l}^{2} \tau_{l}}{\sum_{l=0}^{L-1} \sigma_{l}^{2}}\right)^{2}} .
$$

Here, $\sigma_{l}^{2}$ and $\tau_{l}$ are the average power and delay of the $l$ th path, respectively. We assume that the path power is normalized, i.e., $\sum_{l=0}^{L-1} \sigma_{l}^{2}=1$.

The pilot signal is used for the estimation of channel impulse response (CIR) $H_{d}\left(n_{1}+x, k_{1}+y\right)$ at time $n_{1}+x$ and frequency $k_{1}+y$, as illustrated in Fig. 2. First, the BS estimates the CIR at the pilot signaling as

$$
\hat{H}_{p}(n, k)=\frac{Y(n, k)}{X_{p}(n, k)}=H_{p}(n, k)+\frac{N(n, k)}{X_{p}(n, k)} .
$$

Here, it is assumed that the average power of the pilot symbol $X_{p}(n, k)$ is normalized to one. Then it estimates the CIR at the data signaling by interpolating the CIR at the pilot signaling. Define the mean squared error (MSE) of the channel estimation by

$$
\varepsilon=E\left\{\left|\hat{H}_{d}\left(n_{1}+x, k_{1}+y\right)-H_{d}\left(n_{1}+x, k_{1}+y\right)\right|^{2}\right\}
$$

where $\hat{H}_{d}\left(n_{1}+x, k_{1}+y\right)$ denotes the estimated CIR of the data symbol at time $n_{1}+x$ and frequency $k_{1}+y$. 


\section{Proposed Pilot SignALING}

We consider the adaptation of pilot signal spacing in response to the change of channel characteristics (e.g., Doppler frequency and RMS delay spread). When the channel has a large RMS delay spread, it is desirable to reduce the pilot spacing $d_{f}$ in the frequency domain to achieve desired channel estimation. On the other hand, when the channel has a small RMS delay spread, it can be possible to increase $d_{f}$ without degradation of the channel estimation. Similarly, when the channel experiences high Doppler frequency (i.e., the MS mobility is high), it may be desirable to reduce the pilot spacing $d_{t}$ in the time domain, and vice versa.

We optimize the pilot spacing to minimize the pilot signaling overhead without degradation of the channel estimation in response to the average channel characteristics. We use the MSE as an index of the channel estimation performance. Letting the maximum tolerable MSE be $\Delta E$, the optimum pilot spacing can be determined as

$$
\left(\hat{d}_{t}, \hat{d}_{f}\right)=\arg \max _{\left\{d_{t}, d_{f}: \varepsilon \leq \Delta E\right\}} d_{t} d_{f} .
$$

Thus, the pilot signaling density defined by $1 /\left(d_{t} d_{f}\right)$ can be minimized by maximizing $d_{t} d_{f}$ for a given channel condition.

We consider two channel estimation schemes; linear interpolation (LI) and Wiener interpolation scheme [9], [10]. The former is widely employed in practice due to its processing simplicity and the latter is known to be optimum but requires high computational complexity.

The LI scheme estimates the CIR as

$$
\begin{aligned}
\hat{H}_{d}\left(n_{1}+x, k_{1}+y\right) & =\frac{y}{d_{f}}\left[\hat{H}_{d}\left(n_{1}+x, k_{2}\right)-\hat{H}_{d}\left(n_{1}+x, k_{1}\right)\right] \\
& +\hat{H}_{d}\left(n_{1}+x, k_{1}\right)
\end{aligned}
$$

where

$$
\begin{aligned}
& \hat{H}_{d}\left(n_{1}+x, k_{1}\right)=\frac{x}{d_{t}}\left[\hat{H}_{p}\left(n_{2}, k_{1}\right)-\hat{H}_{p}\left(n_{1}, k_{1}\right)\right]+\hat{H}_{p}\left(n_{1}, k_{1}\right), \\
& \hat{H}_{d}\left(n_{1}+x, k_{2}\right)=\frac{x}{d_{t}}\left[\hat{H}_{p}\left(n_{2}, k_{2}\right)-\hat{H}_{p}\left(n_{1}, k_{2}\right)\right]+\hat{H}_{p}\left(n_{1}, k_{2}\right) .
\end{aligned}
$$

It can be shown that

$$
\begin{aligned}
\hat{H}_{d}\left(n_{1}+x, k_{1}+y\right) & =A \hat{H}_{p}\left(n_{2}, n_{2}\right)+B \hat{H}_{p}\left(n_{1}, k_{2}\right) \\
& +C \hat{H}_{p}\left(n_{2}, k_{1}\right)+D \hat{H}_{p}\left(n_{1}, k_{1}\right)
\end{aligned}
$$

where $\quad A=\frac{x y}{d_{t} d_{f}}, \quad B=\frac{y}{d_{f}}-\frac{x y}{d_{t} d_{f}}, \quad C=\frac{x}{d_{t}}-\frac{x y}{d_{t} d_{f}} \quad$ and $D=\frac{x y}{d_{t} d_{f}}-\frac{y}{d_{f}}-\frac{x}{d_{t}}+1$. By substituting (14) into (10), it can be shown that the MSE of the LI scheme is

$$
\begin{aligned}
\mathcal{E}_{L I}= & 1+\left(1+\sigma_{n}^{2}\right)\left(A^{2}+B^{2}+C^{2}+D^{2}\right) \\
& +2\left(\begin{array}{l}
A B r_{t}\left[d_{t}\right]+A C r_{f}\left[d_{f}\right]+A D r_{t}\left[d_{t}\right] r_{f}\left[d_{f}\right]+ \\
B C r_{t}\left[-d_{t}\right] r_{f}\left[d_{f}\right]+B D r_{f}\left[d_{f}\right]+C D r_{t}\left[d_{t}\right]
\end{array}\right) \\
& -2\left(\begin{array}{l}
A r_{t}\left[d_{t}-x\right] r_{f}\left[d_{f}-y\right]+B r_{t}[-x] r_{f}\left[d_{f}-y\right] \\
+C r_{t}\left[d_{t}-x\right] r_{f}[-y]+D r_{t}[x] r_{f}[y]
\end{array}\right)
\end{aligned}
$$

where $r_{t}[X]=r_{t}\left(X T_{s}\right)$ and $r_{f}[Y]=r_{f}(Y \Delta f)$.

Assuming the use of a two-dimensional Wiener interpolation filter having $N_{\text {tap }}$ taps in both the time and frequency domain, the CIR can be estimated as [10]

$$
\hat{H}_{d}\left(n_{1}+x, k_{1}+y\right)=\left(\mathbf{R}^{-1} \mathbf{P}\right)^{H} \hat{\mathbf{H}}_{\mathbf{p}},
$$

where $\hat{\mathbf{H}}_{\mathrm{p}}$ is an $\left(N_{\text {tap }}^{2} \times 1\right)$-dimensional vector whose each element is the CIR at the pilot signaling, and $\mathbf{R}$ and $\mathbf{P}$ respectively denote the auto-covariance matrix and the crosscovariance matrix of the channel, defined by

$$
\begin{gathered}
\mathbf{R}=E\left\{\hat{\mathbf{H}}_{\mathbf{p}} \hat{\mathbf{H}}_{\mathbf{p}}^{*}\right\} \\
\mathbf{P}=E\left\{\hat{\mathbf{H}}_{\mathbf{p}} \hat{H}_{d}^{*}\left(n_{1}+x, k_{1}+y\right)\right\} .
\end{gathered}
$$

The MSE of the Wiener interpolation filter scheme can be represented as [10]

$$
\varepsilon_{W F}=1-\mathbf{P}^{T} \mathbf{R}^{-1} \mathbf{P}^{*} .
$$

The optimum pilot density of a channel estimation schemes can analytically be determined using the MSE of channel estimation. A pilot spacing set that yields an estimation error less than $\Delta E$ can be determined by using (15) and (19). The optimum pilot density is determined by choosing the element which has the smallest pilot density in the set.

In practice, both the BS and the MS need to share the pilot signaling information (e.g., $d_{t}$ and $d_{f}$ ). Since the average channel characteristics do not vary fast as much as the instantaneous CIR does, it may not be required to frequently change the pilot density, yielding a marginal increase of overhead for the change of pilot density. 
TABLE I

SIMULATION PARAMETERS

\begin{tabular}{cc}
\hline \hline PARAMETERS & Values \\
\hline Carrier frequency & $5.4 \mathrm{GHz}$ \\
Bandwidth & $24 \mathrm{MHz}$ \\
OFDM symbol duration & $20.48 \mathrm{us}$ \\
Guard interval & $5 \mathrm{us}$ \\
Number of subcarriers & 512 \\
Channel & Rayleigh fading \\
Power delay profile & Exponential \\
Tap size of Wiener filter $\left(N_{\text {tap }}\right)$ & 2 \\
\hline \hline
\end{tabular}

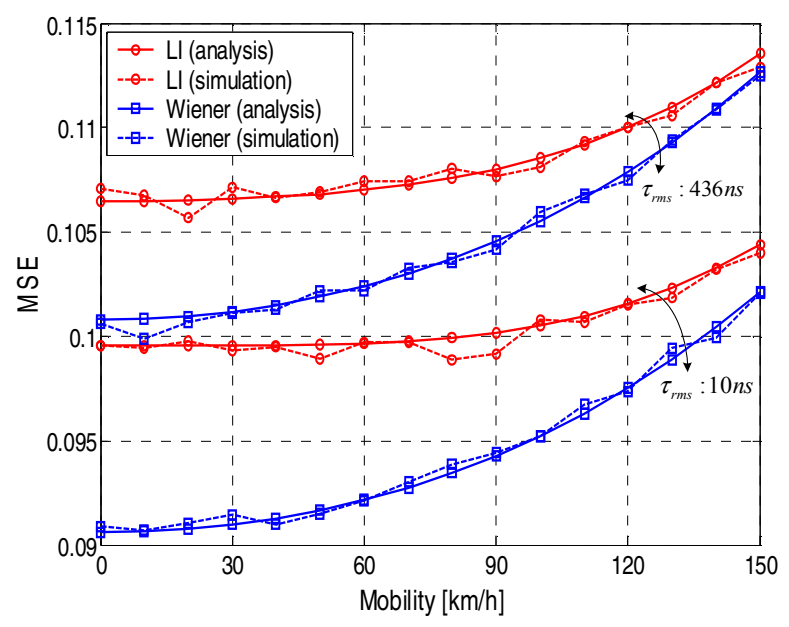

Fig. 3. MSE performance of LI and Wiener channel estimation scheme.

\section{PERFormance EVALUATION}

The performance of the proposed scheme is verified by computer simulation. The simulation parameters are summarized in Table I.

Fig. 3 depicts the MSE of the two interpolation schemes when the pilot spacing is fixed to $d_{t}=8$ and $d_{f}=4$. It can be seen that the MSE increases as the user mobility increases. It can also be seen that the analysis agrees well with the simulation results. Thus the optimum pilot spacing $\hat{d}_{t}$ and $\hat{d}_{f}$ can be determined by using (15) or (19).

Fig. 4 depicts the pilot spacing to keep the MSE less than $\Delta E$ in terms of the user mobility and RMS delay. It can be seen that as the selectivity of the channel decreases, the pilot density needs to be increased. It can also be seen that the Wiener filter requires less pilot signal than the LI filter and that the optimum pilot spacing in the time domain largely depends on the pilot spacing in the frequency domain, and vice versa. Therefore, it is desirable to determine the pilot density jointly considering in the time and frequency domain.

Fig. 5 depicts the minimum pilot density that provides a

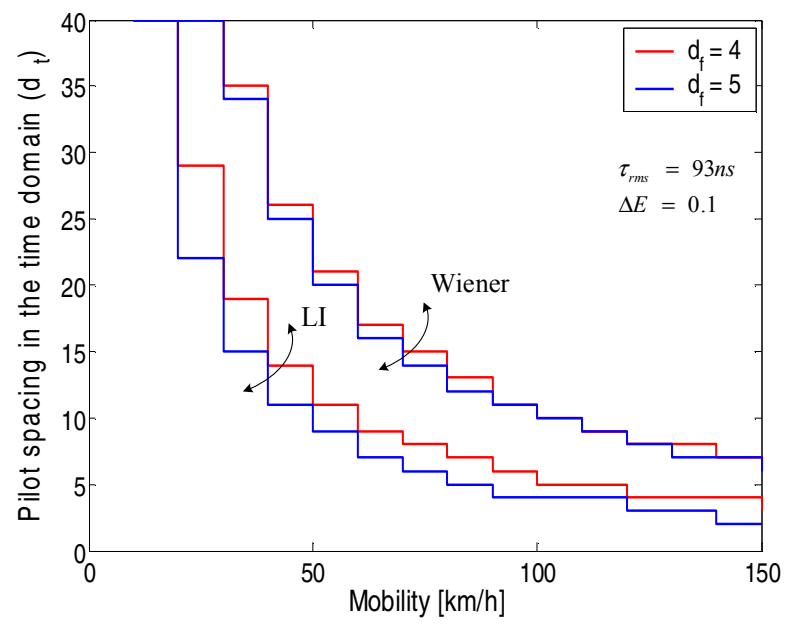

(a) Maximum pilot spacing in the time domain

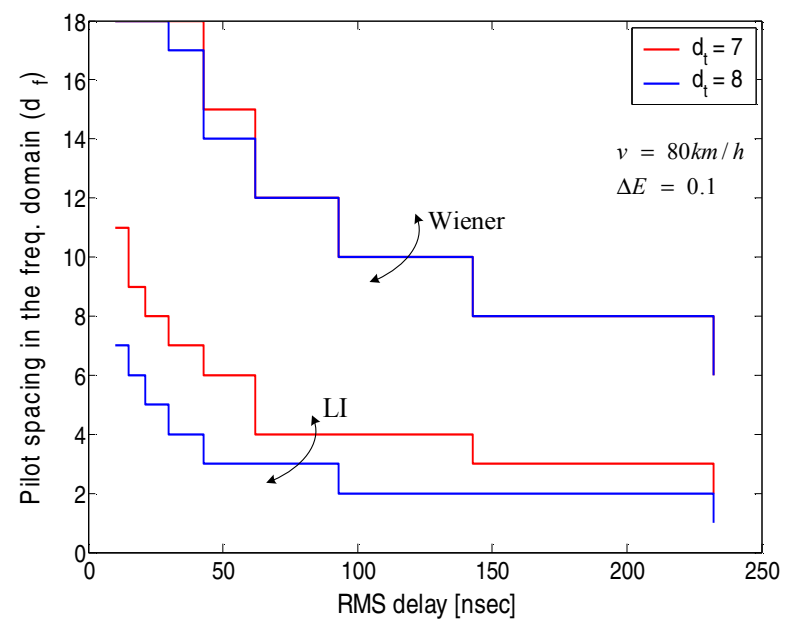

(b) Maximum pilot spacing in the frequency domain

Fig. 4. Maximum pilot spacing when LI and Wiener filter is used.

MSE less than a given $\Delta E$ associated with the mobility and RMS delay spread. It can be seen that the pilot density increases as the mobility and/or the RMS delay spread increase. It can also be seen that the Wiener filter scheme requires a pilot density lower than the LI filter scheme for a given threshold value $\Delta E$ at the expense of increased implementation complexity.

Table II summarizes the reduction of pilot overhead by the proposed scheme for $\Delta E=0.1$, where $\left(d_{t}, d_{f}\right)$ denotes the spacing of the pilot signal in the time and frequency domain. For ease of implementation, the channel condition is classified into three groups, I, II and III. The thresholds for the classification are $\langle 150,10\rangle,\langle 300,43\rangle$ and $\langle 750,143\rangle$, 


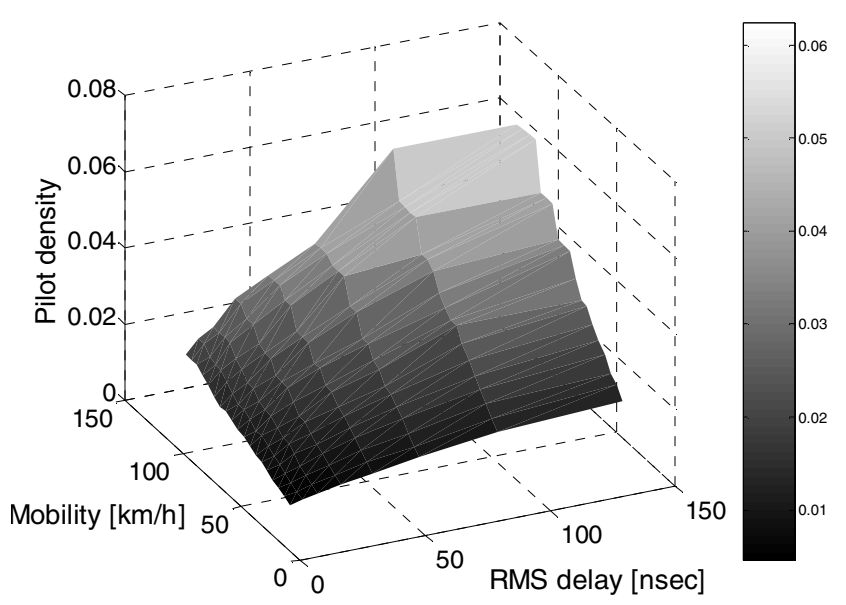

(a) LI

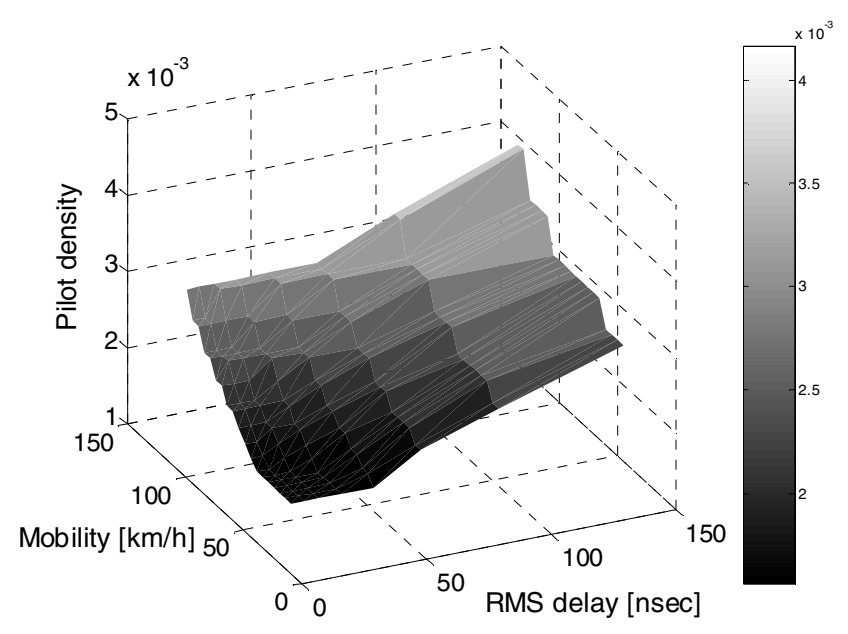

(b) Wiener filter

Fig. 5. Optimum pilot density for $\Delta E=0.1$.

where $\langle x, y\rangle$ denotes Doppler frequency $x(\mathrm{~Hz})$ and RMS delay spread $y$ (nsec). We assume that the conventional scheme using a fixed pilot density that can support the worst channel environment (i.e., group III). It can be seen that the proposed scheme can considerably reduce the pilot density by adapting the pilot spacing according to the channel condition. For example, when MSs have a distribution of $50 \%$ for group I, $30 \%$ for group II and $20 \%$ for group III, the proposed scheme can reduce the pilot overhead to approximately $1 / 3$ compared to the conventional fixed pilot scheme.
TABLE II

Pilot Density of The Proposed Scheme

\begin{tabular}{c|cccc}
\hline \hline \multicolumn{1}{c}{} & & Group I & Group II & Group III \\
\hline \multirow{5}{*}{ LI } & Fixed pilot density & 0.0833 & 0.0833 & 0.0833 \\
& $\left(d_{t}, d_{f}\right)$ & $(3,4)$ & $(3,4)$ & $(3,4)$ \\
& Adaptive pilot density & 0.0046 & 0.0278 & 0.0833 \\
& $\left(\hat{d}_{t, L I}, \hat{d}_{f, L I}\right)$ & $(18,12)$ & $(6,6)$ & $(3,4)$ \\
\hline \multirow{5}{*}{ Wiener } & $\begin{array}{c}\text { Fixed pilot density } \\
\left(d_{t}, d_{f}\right)\end{array}$ & 0.0278 & 0.0278 & 0.0278 \\
& Adaptive pilot density & 0.0022 & 0.0089 & 0.0278 \\
& $\left(\hat{d}_{t, W F}, \hat{d}_{f, W F}\right)$ & $(30,15)$ & $(8,14)$ & $(6,6)$ \\
\hline \hline
\end{tabular}

\section{CONCLUSIONS}

In this paper, we have proposed an adaptive pilot signaling scheme in the uplink of an OFDM based wireless system. The proposed scheme adjusts the pilot density by exploiting the average channel characteristics. The minimum density of the pilot signal is analytically adjusted using the MSE of channel estimation in response to the change of channel condition. The simulation results show that the proposed scheme can significantly reduce the pilot signaling overhead while providing desired channel estimation error.

\section{REFERENCES}

[1] S. Hara and R. Prasad, Multicarrier Techniques for 4G Mobile Communications, Artech House, first edition, 2003.

[2] H. Sampath, S. Talwar, J. Tellado, V. Erceg, and A. Paulraj, "A fourthgeneration MIMO-OFDM broadband wireless system: design, performance, and field trial results," IEEE Commun. Mag., vol. 40, no. 9, pp. 143-149, Sept. 2002.

[3] F. Tufvesson and T. Maseng, "Pilot assisted channel estimation for OFDM in mobile cellular systems," in Proc. IEEE VTC'97, pp. 1639-1643, May 1997.

[4] M. Sandell and O. Edfors, "A comparative study of pilot-based channel estimators for wireless OFDM," Research Report TULEA, vol. 31, Sept. 1996.

[5] S. Coleri, M. Ergen, A. Puri and A. Bahai, "Channel estimation techniques based on pilot arrangement in OFDM systems," IEEE Trans. Broadcasting, vol. 48, no. 3, Sept. 2002.

[6] Ji-Woong Choi and Yong-Hwan Lee, "Design of the optimum pilot pattern for channel estimation in OFDM systems," in Proc. IEEE Globecom'04, pp. 3661-3665, Dec. 2004.

[7] H. Arslan and T. Yucek, "Delay spread estimation for wireless communication systems," in Proc. IEEE ISCC'03, pp. 280-287, June 2003.

[8] T. Rappaport, Wireless Communications, Prentice Hall, second edition, 2001.

[9] Jihyung Kim, Jeongho Park and Daesik Hong, "Performance analysis of channel estimation in OFDM systems," IEEE Signal Process. Lett., vol. 12, no. 1, pp. 60-62, Jan. 2005.

[10] P. Hoeher, S. Kaiser and P. Roberston, "Two-dimensional pilot-symbol aided channel estimation by Wiener filtering," in Proc. IEEE ICASSP'97, pp. 1845-1848, Apr. 1997. 